\title{
SCATTERING OF $\mu$ MESONS BY CARBON
}

\author{
A. Citron, C. Delorme, D. Fries, L. Goldzahl (*), J. Heintze, E. G. Michaelis, C. Richard (**) \\ and $H$. Øverås
}

CERN, Genève

(presented by A. Citron)

Comparison of $\mu$ meson scattering and electron scattering provides a means of studying the behaviour of the $\mu$ vertex alone, at a momentum transfer imposed by the experimental conditions. With this aim in mind we have scattered negative $\mu$ mesons from the CERN SC at 240 and $180 \mathrm{MeV} / \mathrm{c}$ incident momentum and through angles between 30 and $72.5^{\circ}$. In this way four momentum transfers up to $q^{2}=1.5 \mathrm{fermi}^{-2}$ were reached. Some details about these measurements have already been published ${ }^{1)}$. I shall mainly give some complementary information.

As a scatterer we have used carbon. It has sometimes been suggested that the experiment would be much more meaningful if hydrogen was used. In our opinion the choice of the scatterer is irrelevant in principle, since its behaviour drops out in the comparison. It is necessary, however, that complete information is available about the electron scattering in the substance chosen. As long as the first Born approximation is valid, this means in general that we must know two form factors for the elastic scattering and two for the inelastic scattering into each excited level. In the case of hydrogen we have, in our energy range, no inelastic scattering, which simplifies the situation. We need, however, two form factors to describe the elastic scattering. In the case of carbon, a spin zero nucleus, a single form factor describes the elastic scattering. The scattering into some of the inelastic levels still requires, in principle, two form factors. It turns out, however, that the contribution of the magnetic form factors is negligible in our $q$ range. Therefore, we obtain all the necessary information at a certain $q$ by extending the integration over the momentum of the scattered particle, not only over the elastic peak, but also over as many of the inelastic peaks as contribute to the $\mu$ scattering in our apparatus. We took this information from published data obtained in Stanford and Orsay. Recently Professor Bishop at Orsay was kind enough to measure some check points, both elastic and inelastic, for us. The results confirm the form factors we used within the precision required. A nucleus of much higher $Z$ has the disadvantage of a bigger multiple-scattering contribution (which never exceeded $12 \%$ in our case). Moreover, the validity of the first Born approximation is less certain. In our case, this validity is checked in the electron scattering and also in our own experiment, where the same momentum transfer is reached with two different incident momenta.

In view of the relatively small flux of $\mu$ mesons which we can obtain $\left(2 \times 10^{4} / \mathrm{sec}\right)$, our apparatus had to accept a large solid angle. In order to make a precise comparison with electron scattering possible, the momentum and angular distributions, although wide, must be well defined. Finally there must be a high degree of discrimination against $\pi$ meson scattering, since the corresponding cross-sections are much higher.

The apparatus is shown in Fig. 1. Scattered particles were required to stop in counter 7 . This range requirement provides some discrimination against $\pi$ mesons and limits the contribution from inelastic scattering. The stopped $\mu$ mesons were then required to give a delayed coincidence within a $5 \mu \mathrm{sec}$ gate, due to the decay electron. This $\mu$ signature provides a good discrimination against $\pi$ mesons.

(*) Laboratoire de Synthèse Atomique, Ivry.

(**) Laboratoire de Physique Nucléaire, Unversitè de Grenoble, Grenoble. 


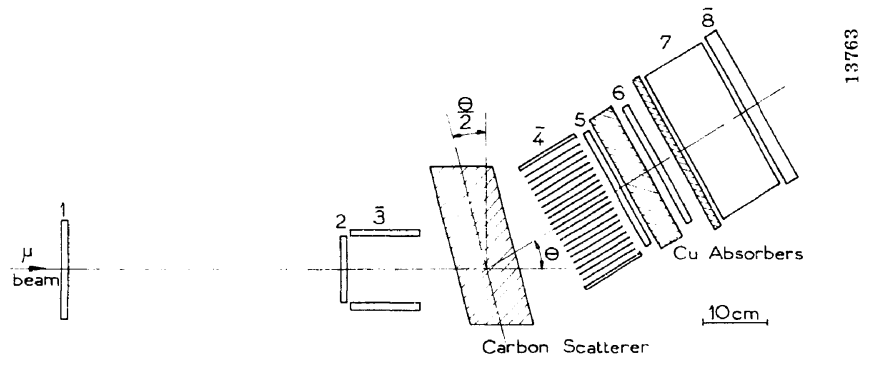

Fig. 1 Drawing of the apparatus. $\mu$-meson event $12 \overline{3} \overline{4} 567 \overline{8}+7$ delayed. $\overline{4}$ is a "Venetian blind" counter, providing angular selection. $\overline{3}$ is an anticoincidence frame, which can also be converted into a Venetian blind by inserting scintillator sheets. In this way, and by increasing the number of sheets in $\overline{4}$, the angular resolution can be improved.

A second gate allowed background subtraction. The telescope was first exposed to the direct beam to determine the detection efficiency. Then it was tilted and the scattering rates were recorded. In this way, absolute scattering probabilities were measured.

These scattering probabilities required a correction for events, where a $\pi$ meson is scattered and decays in flight, transmitting to the $\mu$ meson an energy such that it is detected in counter 7. The correction for these events was obtained by repeating the scattering experiment in a beam artificially polluted with $\pi$ mesons. If we introduce the quantities given in

TABLE I

\begin{tabular}{|l|c|c|}
\hline & pure beam & polluted beam \\
\cline { 2 - 3 } contamination & $n \ll 1$ & $N$ \\
counting rate & $\gamma$ & $\Gamma$ \\
\hline
\end{tabular}

Table I then the correction to be applied to the $\mu$ scattering rates is given by

$$
\gamma_{\pi}=\frac{n}{N}[\Gamma-\gamma(1-N)]
$$

The quantity $n / N$, the ratio of the $\pi$ contamination in the two types of beams, was determined by measuring range curves in the conventional arrangement for differential range curves, but requiring a large light output in the counter where the particles stop. This large light output is due to a star, and is thus characteristic for a $\pi$ meson stopping. This method allows us to measure the momentum distribution of $\pi$ mesons in the presence of a large number of $\mu$ mesons ${ }^{2}$. The $\pi$ momentum distributions in the two types of beam look somewhat different. The ratio of the areas below these curves gives the relative purity. Since, however, the decay in flight and detection probability is a function of momentum, it is more correct to weigh these distributions first with this probability and then to integrate them. The unweighed $n$ corresponds to a purity of $2 \%$, the weighed to about $3 \%$.

The scattering rates corresponding to a heavy electron have been calculated using the Mott crosssection, the carbon form factors and the momentum, angular and spatial distribution of the $\mu$ beam. The momentum distribution was obtained from a precise range curve. In this case we demanded the delayed coincidence signal in the counter where the particles stop, so that only $\mu$ stops were recorded. This is essential in the wings of the distribution.

Fig. 2 shows the measured and calculated scattering rates. It is seen that no significant deviation from the calculated rates is measured. This becomes more apparent on Fig. 3, where the ratio between measured and calculated rates is plotted as a function of $q^{2}$. We see that the points taken at different incident

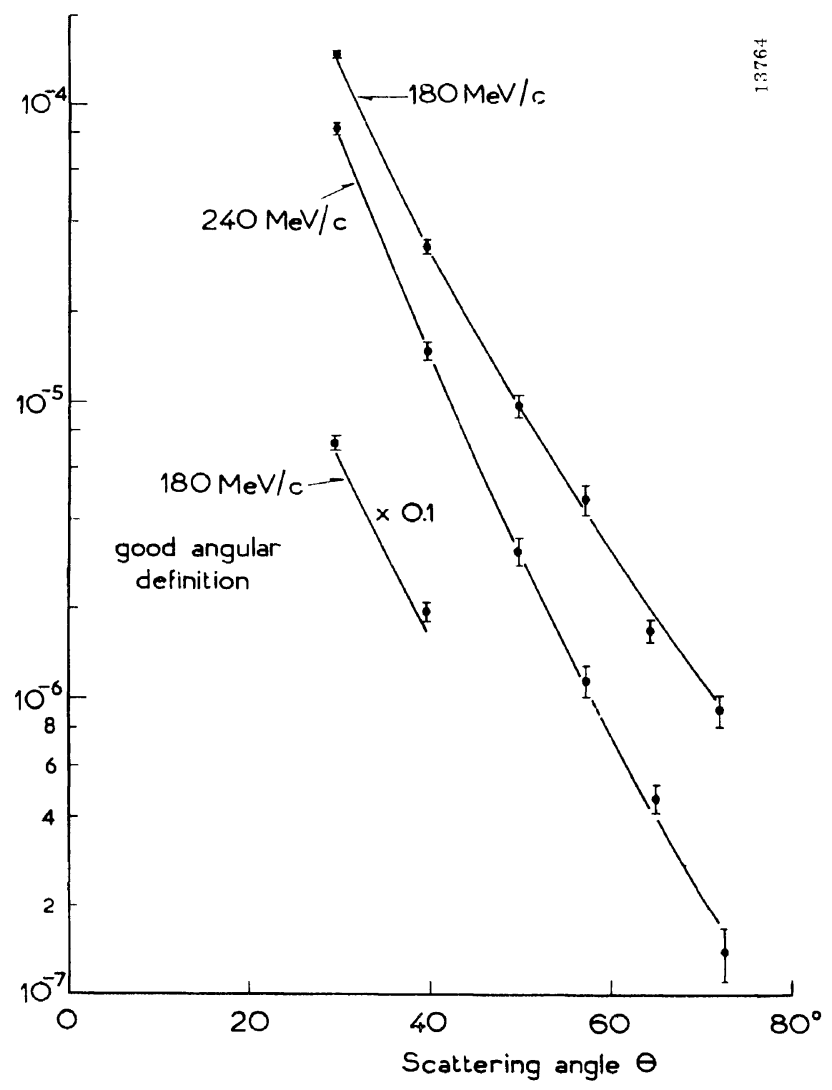

Fig. 2 Scattering at angle $\theta$, divided by scattering rates at angle $0^{\circ}$, as a function of the telescope angle $\theta$. Observed ratios are indicated by dots, calculated ratios by the drawn lines. Errors indicated are statistical only. 




Fig. 3 Ratio $R$ of observed to calculated rates as a function of the square of momentum transfer. The dashed lines on either side of the axis $R=1$ correspond to the estimate of the systematic error. The error bars represent statistical errors. Lines labelled with values of $\Lambda^{-1}$ replace the line $R=1$, if the calculated rates are based on a structure of the $\mu$ vertex described by this cut-off. momenta merge very nicely. The points scatter normally around the straight line which corresponds to the ratio being unity everywhere. A $\chi^{2}$ test on this hypothesis gives $P=50 \%$. Included on the diagram are also lines which replace the straight line mentioned under the hypothesis that the $\mu$ vertex is altered, due to a structure of the $\mu$ meson in the conventional way with values of the cut-off length $\Lambda^{-1}$ as marked at each line. We can then ask which cut-off we can exclude with which confidence. The analysis yields:

$\Lambda^{-1} \leqq 0.18$ fermi for $70 \%$ confidence (about one standard deviation),

$\Lambda^{-1} \leqq 0.27$ fermi for $95 \%$ confidence (about two standard deviations).

Within these limits the $\mu$ meson behaves just like a heavy electron.

\section{LIST OF REFERENCES}

1. A. Citron, C. Delorme, D. Fries, L. Goldzahl, J. Heintze, E. G. Michaelis, C. Richard and H. Øverås. Phys. Letters 1, 175 (1962).

2. A. Citron, C. Delorme, D. Fries, J. Heintze, E. G. Michaelis, H. Øverås and Yu. Shcherbakov. Nucl. Instr. and Methods 15, 121 (1962).

\section{DISCUSSION}

WOLFENSTEIN: What is the situation with respect to inelastic scattering. You resolve the inelastic scattering?

CITRON: We know the detection probability as a function of the final muon energy. It is $100 \%$, say, in the elastic peak, because things are centred that way, and then it falls off according to a function which is given by our momentum spread and the range requirement. So we have to weigh the various levels with the detection probability. We do include the 3 first levels, i.e., the 4.4. and 7.6 and 9.6 MeV levels. The others are sufficiently suppressed, so that we can forget about them.

WOLFENSTEIN: And you include that in your analysis?

Citron: Yes. The inelastic levels make a substantial contribution (about $40 \%$ ) to the last point with the largest momentum transfer, and rather less for all other points, because the elastic form factor falls down like a stone, whereas the others are more or less level.

TELEgDi: There is one point I do not understand, and that is how do you know how strongly these levels will be populated, unless you assume ahead of time they will be populated as in the electron experiments. This must be postulated in this respect, say the behaviour is identical or has some presumed magnitude for the higher levels' inelastic part.

Citron: As I understand, what you say is this: how do you know that the $\mu$ mesons and the electrons have the same role in exciting these inelastic levels? I think the answer is that the whole philosophy of the experiment is that we assume everything is as in the electron case, and we see whether we can get away with that assumption. If there were a deviation, admittedly, one would have to ask if it were due to the elastic or to the inelastic scattering. For the electromagnetic interaction of the $\mu$ meson the difference between elastic and inelastic scattering is not really very fundamental.

PANOFSKY: I believe I can answer Telegdi's question more definitely. One can show in first Born approximation that one can write for the inelastic scattering a general expression for the cross-section in terms of two form factors which are not only functions of $q^{2}$ but also functions of $\Delta E$, but where the mass does not appear explicitly.

CITRON: I suspect that Telegdi meant that if there was some mysterious interaction other than electromagnetic between $\mu$ mesons and nucleons, then this may act in a different way in the inelastic than in the elastic case. 
BERMAN: What is the error introduced by the angular resolution of the incident beam?

Citron: I do not think there is much of an error introduced there. The angular resolution is given by folding the angular spread of the incident beam with the triangular angular resolution function of our apparatus, which is a Venetian blind, i.e. a set of thin scintillator sheets in anti-coincidence. This folded resolution we measure directly by eliminating the target and exposing the Venetian blind at various angles to the incident beam. We integrate over it to find the theoretical counting rate. The uncertainty is given by the fact that this angular distribution is not quite independent of location, by the precision in the setting of angles etc., but these errors are at most 1 or $2 \%$. Incidentally, an experimental check of this aspect is built into our experiment, since some points were repeated at improved angular resolution, obtained by adding sheets in the Venetian blind counters $\overline{3}$ and $\overline{4}$.

\title{
MESONIC ATOM PROCESSES IN HYDROGEN AND DEUTERIUM AND THE MUON CATALYSIS OF FUSION REACTIONS
}

\author{
V. P. Dzhelepov, M. Friml, S. S. Gershtejn, Yu. V. Katyshev, V. I. Moskalev, P. F. Yermolov
}

Joint Institute for Nuclear Research, Dubna

(presented by V. P. Dzhelepov)

A study of properties of some fundamental processes taking place with muon participation (nuclear capture of muons by protons, muon catalysis of fusion reactions of hydrogen isotopes etc.) call for the knowledge of quite a number of features of

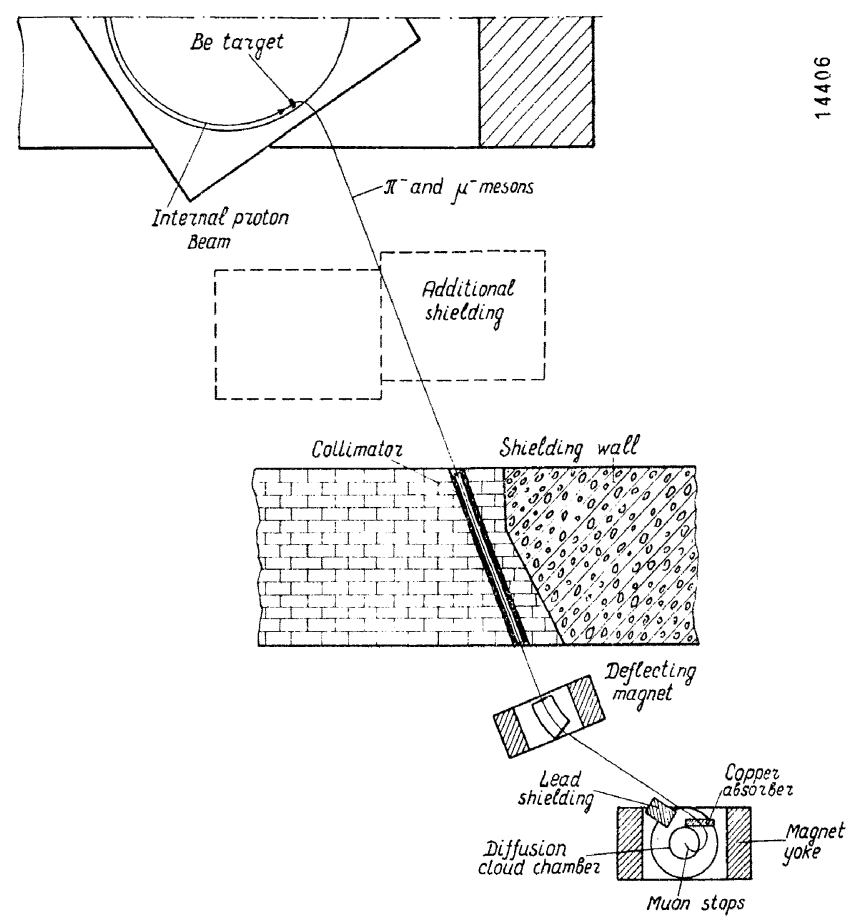

Fig. 1 Experimental arrangement.

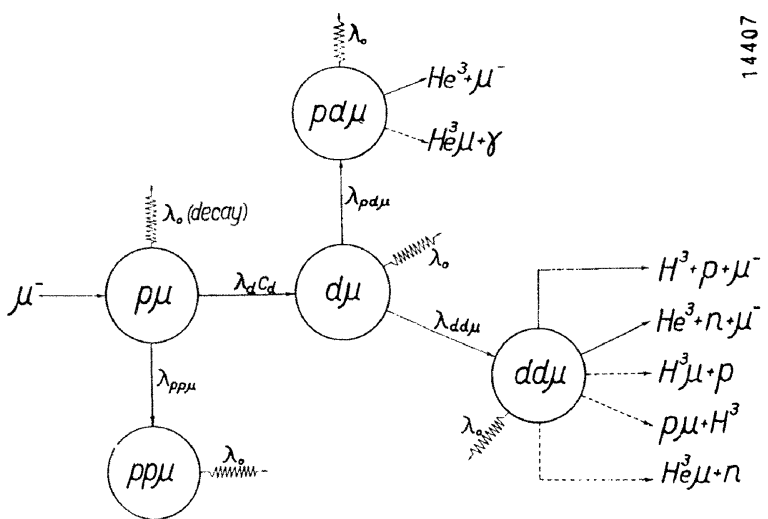

Fig. 2 Diagram of mesonic atom and mesonic ion formation and the catalysis of possible nuclear fusion reactions by negative muons in hydrogen and deuterium mixtures.

meso-atomic and meso-molecular phenomena occurring in hydrogen and deuterium ${ }^{1,2)}$. These phenomena are interesting in themselves since by investigating mesonic atoms one obtains information on the behaviour of these peculiar systems. In this connection a number of corresponding experiments have been carried out with a high pressure diffusion chamber (40 $\mathrm{cm}$ in diameter) in a magnetic field of 7,000 Oe at the synchro-cyclotron of the Joint Institute for Nuclear Research (the experimental arrangement is shown in Fig. 1). Processes shown by solid lines in Fig. 2 have been studied. 